\title{
Gamma-ray emission from star-forming complexes observed by MAGIC: The cases of W51 and HESS J1857+026
}

\author{
I. Reichardt ${ }^{1, \text { a }}$, E. Carmona ${ }^{2}$, S.R. Gozzini ${ }^{3}$, S. Klepser ${ }^{4,5}$, J. Krause ${ }^{6,7}$, and V. Stamatescu ${ }^{4,8}$ on behalf of the MAGIC \\ Collaboration \\ 1 Università di Padova and INFN, 35131 Padova, Italy \\ 2 Centro de Investigaciones Energéticas, Medioambientales y Tecnológicas, 28040 Madrid, Spain \\ 3 Deutsches Elektronen-Synchrotron (DESY), 15738 Zeuthen, Germany \\ 4 IFAE, Campus UAB, 08193 Bellaterra, Spain \\ 5 Now at Deutsches Elektronen-Synchrotron (DESY), 15738 Zeuthen, Germany \\ 6 Max-Planck-Institut für Physik, 80805 München, Germany \\ 7 Now at Astroparticule et Cosmologie (APC), Université Paris 7 Denis Diderot, 75205 Paris Cedex 13, France \\ 8 Now at School of Chemistry \& Physics, University of Adelaide, Adelaide 5005, Australia
}

\begin{abstract}
Massive star-forming regions assemble a large number of young stars with remnants of stellar evolution and a very dense environment. Therefore, particles accelerated in supernova remnants and pulsar wind nebulae encounter optimal conditions for interacting with target material and photon fields, and thus produce gamma-ray emission. However, observations are challenging because multiple phenomena may appear entangled within the resolution of current gamma-ray telescopes. We report on MAGIC observations aimed to understand the nature of the emission from the star-forming region W51 and the unidentified source HESS J1857+026. While gamma-ray emission from W51 is dominated by the interaction of the supernova remnant W51C with dense molecular clouds, HESS J1857+026 is associated to the pulsar wind nebula from PSR J1856+0245. However, an additional source is resolved north of HESSJ1857+026, with sufficient separation to determine that it cannot be powered by the same pulsar. We search for multiwavelength data to determine the origin of the new source.
\end{abstract}

\section{Introduction}

MAGIC are two imaging atmospheric Cherenkov telescopes characterized by its $\sim 50 \mathrm{GeV}$ threshold, the lowest achieved in ground-based stereo observations of veryhigh-energy (VHE) gamma rays. The best performance of MAGIC is achieved above $300 \mathrm{GeV}$ [1], where the current sensitivity ${ }^{1}$ is $0.56 \%$ the flux of the Crab Nebula; the angular resolution is $0.07^{\circ}$; and the energy resolution is about $16 \%$.

The science cases for MAGIC include the observation of Galactic sources of VHE gamma rays: to resolve the multiple components that may overlap along the line of sight; to understand the physical processes that produce the observed gamma-ray emission; and to assess the contribution that these sources may have to the Galactic cosmic rays. In this contribution, we discuss the particular cases of W51 [2] and HESSJ1857+026 [3]. These sources were observed for 53 and 29 hours (effective time, respectively) in 2010 and 2011. The configuration and performance of the telescopes at that time is described in [4].

\footnotetext{
a e-mail: ignasi.reichardt@pd.infn. it

${ }^{1}$ Sensitivity is defined as the minimum flux from a point-like source to be detected with a significance of $5 \sigma$ in 50 hours of observations.
}

\section{The supernova remnant W51C in W51}

\subsection{Multiwavelength context}

W51 is a very massive giant molecular complex composed by the star-forming regions W51A and $\mathrm{W} 51 \mathrm{~B}$, and the supernova remnant (SNR) W51C. In [5], a very comprehensive study of the region where $\mathrm{W} 51 \mathrm{~B}$ and W51C overlap, confirmed that there is physical interaction between the two components ${ }^{2}$. Some remarkable features of the interaction region are: the presence of $1720 \mathrm{MHz}$ $\mathrm{OH}$ masers; the non-thermal radio emission surrounding the HII region G49.2-0.2 (which is engulfed by the SNR); and the discovery of a non-thermal, unresolved source W51B_NT.

The X-ray emission from W51C is dominated by thermal emission from shocked material in the SNR interior [10]. However, a few hard, non-thermal sources have been discovered [11]. Notably:

- The compact source CXOJ192318.5+1403035 [12] is a presumed pulsar wind nebula (PWN), with energetics compatible with being generated by the compact object resulting from the W51C progenitor.

- Towards the northwest of W51C, precisely where the SNR interacts with W51B, the diffuse source

\footnotetext{
2 The W51B/C interaction was already quite clear from [6-8], but some controversy was recently raised by [9].
}

This is an Open Access article distributed under the terms of the Creative Commons Attribution License 4.0, which permits unrestricted use, distribution, and reproduction in any medium, provided the original work is properly cited. 


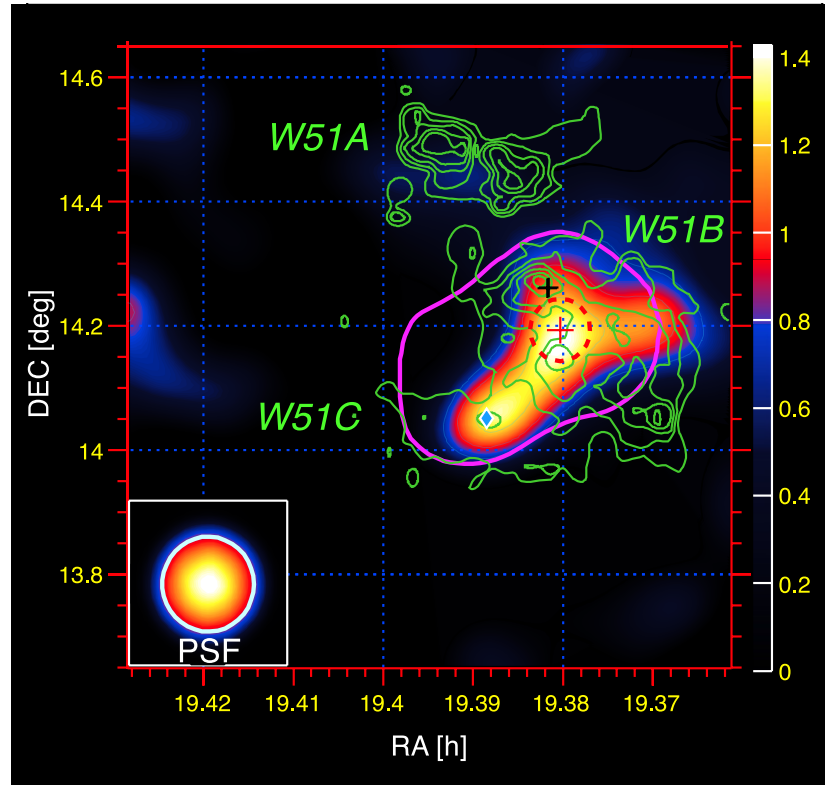

Figure 1. Relative flux map for energies above $1 \mathrm{TeV}$ observed by MAGIC. Green contours represent the $21 \mathrm{~cm}$ radio continuum emission is shown from [6]. The blue diamond represents the position of CXOJ192318.5+140305 and the black cross the position of the $\mathrm{OH}$ maser emission. The red dashed ellipse represents the region where $\mathrm{W} 51 \mathrm{~B}$ and $\mathrm{W} 51 \mathrm{C}$ interact. The gamma-ray emission detected by Fermi/LAT above $1 \mathrm{GeV}$ is depicted by the pink contour. In addition, the test statistic (TS) contours for MAGIC (cyan) are shown, starting at 3 and increasing by one per contour.

HX3-west has uncertain nature. It is not excluded to be an additional PWN [13]; but it can also be the result from collective effects of OB stars in W51B, or due to synchrotron emission from $\sim$ tens of $\mathrm{TeV}$ electrons accelerated by W51C [14].

A gamma-ray source in W51 was discovered from early Fermi/LAT data [15]. The modeling of the spectral energy distribution (SED) suggested that the gamma-ray emission is of hadronic origin, with an extreme luminosity of $\sim 10^{36} \mathrm{erg} / \mathrm{s}$. Together with the Fermi/LAT detection, a coincident VHE detection was reported by H.E.S.S. [16]; and a hint of multi-TeV emission was reported by MILAGRO [17]. Recently, an updated analysis of the Fermi/LAT, data [18] has evidenced the existence of lowenergy roll-off in the gamma-ray spectrum of W51C, usually attributed to the pion production threshold [19].

\subsection{MAGIC observations of W51}

The MAGIC observations campaign on W51 resulted in a highly significant $(11 \sigma)$ detection of a VHE source, precisely at the $\mathrm{W} 51 \mathrm{~B} / \mathrm{C}$ interaction region described above. The morphology of the emission above $1 \mathrm{TeV}$ (Figure 1) suggests a small contribution from the PWN CXOJ192318.5+1403035, but cannot be statistically established as an additional source. The total gamma-ray flux is about $3 \%$ that of the Crab Nebula, and the spectrum follows a power law with index $\Gamma=2.58 \pm 0.07_{\text {stat }} \pm$ $0.22_{\text {sys }}$ between $58 \mathrm{GeV}$ and $5.5 \mathrm{TeV}$.
We fitted SED of W51C with a one-zone model, from radio [20] to VHE, including the Fermi/LAT flux points from [15]. Already before knowing the recent results from [18], we realized that a model where hadronic emission dominates over leptonic processes was strongly favored, and the flux level recently measured around $100 \mathrm{MeV}$ was correctly predicted. Under the assumptions of this model, W51C accelerates protons up to at least $120 \mathrm{TeV}$, with a remarkable efficiency in converting kinetic energy into accelerated particles of about $16 \%$.

\section{The unidentified VHE source HESSJ1857+026}

\subsection{Previous observations}

HESSJ1857+026 was discovered in the H.E.S.S. Galactic Plane Survey and initially classified as unidentified [21]. Shortly after, the newly discovered pulsar PSR J1856+0245 and the nebula around it [22] became the most plausible counterpart for the VHE source. As a PWN, HESSJ1857+026 is peculiar in the sense that it is one of the few such objects detected by Fermi/LAT [23], while no gamma-ray pulsations are detected from PSR J1856+0245. It is interesting to note that, to fit the Fermi/LAT data, two electron populations must be invoked. Moreover, a source slightly north of PSR J1856+0245 was introduced in the Fermi/LAT source model as an analysis refinement. This additional source was not significantly detected above $300 \mathrm{MeV}$ (significance is $3.6 \sigma$ ), and is considered a background source in [23].

\subsection{MAGIC observations of HESSJ1857+026}

MAGIC detected the extended gamma-ray emission from HESSJ $1857+026$ with $12 \sigma$ significance above $150 \mathrm{GeV}$. The spectrum follows a power law with index $\Gamma=2.16 \pm$ $0.07_{\text {stat }} \pm 0.15_{\text {sys }}$, and the flux is $16 \%$ that of the Crab Nebula at $1 \mathrm{TeV}$. The MAGIC measurement smoothly connects the spectral points from Fermi/LAT with H.E.S.S., thus resolving a peak in the SED. At estimated energies between $300 \mathrm{GeV}$ and $1 \mathrm{TeV}$, the position and morphology of the source is compatible with that seen with H.E.S.S. However, above $1 \mathrm{TeV}$ (Fig. 2) two statistically significant components are resolved, MAGIC J1857.2+0263 and MAGIC J1857.6+0297. While MAGIC J1857.2+0263 is likely to be associated to the PWN of PSR J1856+0245, the nature of MAGIC J1857.6+0297 is not known. Being PSR J1856+0245 at an estimated distance of $9 \mathrm{kpc}$, the angular separation between the two MAGIC sources ensures that they are not produced by the same electron population.

The ${ }^{13} \mathrm{CO}(J=1 \rightarrow 0)$ line emission [24] in the radial velocity range 53.76 to $56.11 \mathrm{~km} / \mathrm{s}$ displays a shell-like structure coincident with MAGIC J1857.6+0297 (Fig. 3). This feature is inside the molecular cloud G036.59-00.06 (kinematic distance of $3.6 \mathrm{kpc},[25]$ ), and could be a wind-blown bubble. We suggest that possible origins of the gamma-ray emission from MAGIC J1857.6+0297 are: a PWN whose progenitor star created a cavity in the cloud prior to its explosion [26]; or particle acceleration 


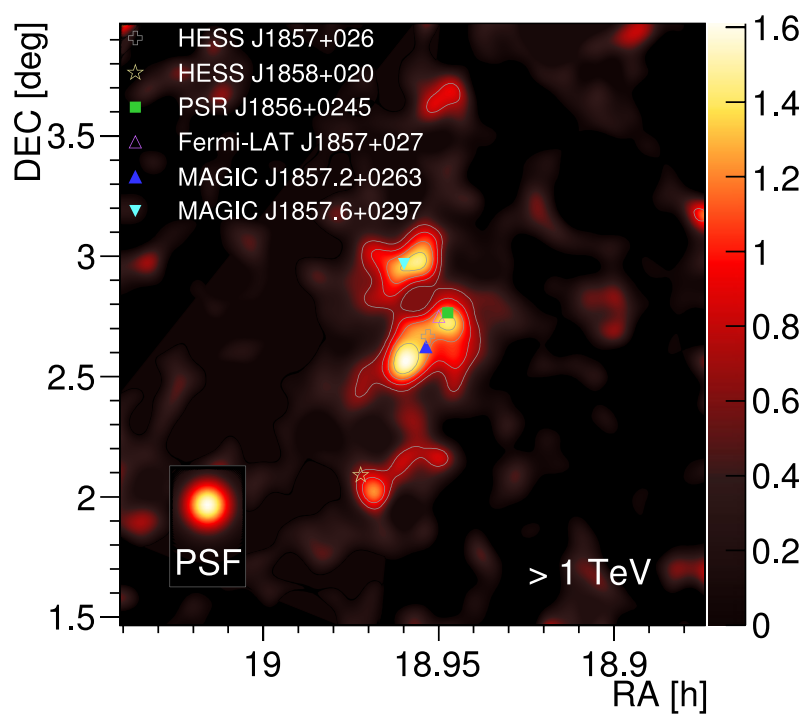

Figure 2. MAGIC gamma-ray relative flux map for events with estimated energy $>1 \mathrm{TeV}$ overlaid with TS contours in steps of 1 , starting at 3 . The positions of the two resolved components, MAGIC J1857.2+0263 and MAGIC J1857.6+0297, are indicated with blue triangles together with the position of HESSJ1857+026 determined by Fermi/LAT [23] and H.E.S.S. [21]. For reference, it is also shown the position HESS J1858+020, another unidentified source not discussed in this article.

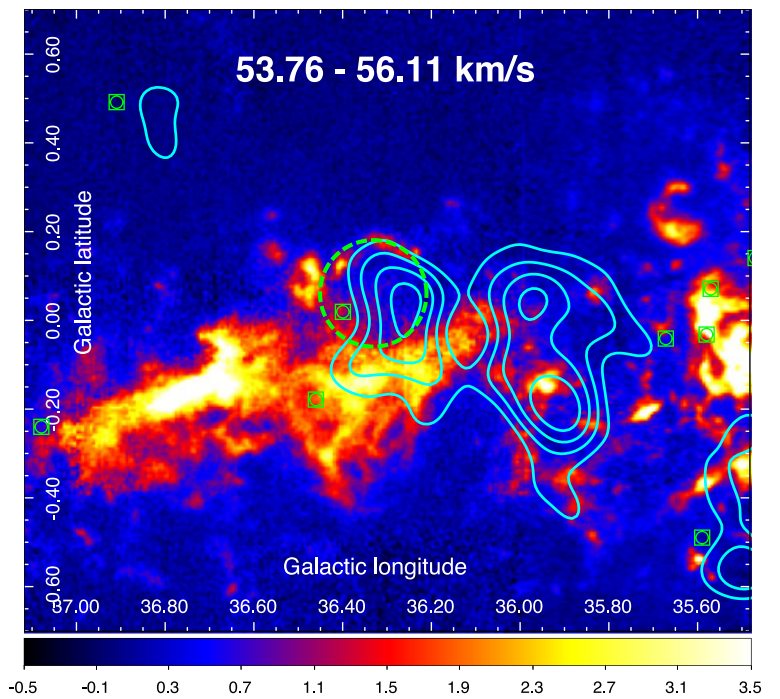

Figure 3. ${ }^{13} \mathrm{CO}(J=1 \rightarrow 0)$ line emission integrated intensity (in units of $\mathrm{K} \mathrm{km} \mathrm{s}^{-1}$ ) in the vicinity of HII region U36.40+0.02 $\left(\mathrm{V}_{\mathrm{LSR}}=53.3 \mathrm{~km} \mathrm{~s}^{-1}\right)$ and of the cataloged clouds G036.59-00.06 $\left(\mathrm{V}_{\mathrm{LSR}}=53.49 \mathrm{~km} \mathrm{~s}^{-1}\right)$ and G036.74-00.16 $\left(\mathrm{V}_{\mathrm{LSR}}=55.19 \mathrm{~km} \mathrm{~s}^{-1}\right)$. The cyan contours indicate the MAGIC $>1 \mathrm{TeV}$ TS levels from Fig. 2 in steps of 1 , starting at 3 . The circle-square green markers indicate HII regions in the catalog of [29]. The dashed green circle indicates the position of a possible cavity in the molecular gas.

based on the combined effects of strong stellar winds from massive stars, or outflows from massive protostars $[27,28]$.

\section{Conclusions}

The VHE observations of W51 poses the question of how a relatively old SNR, whose shock should have been slowed down by the dense environment long ago, still accelerates protons so efficiently, up to energies above $100 \mathrm{TeV}$. Factors contributing to the extreme luminosity of W51C could be presence of HII regions, or collective effects from early-type stars and protostars near the SNR shock. If gamma-ray emission enhancement also occurs in other regions where intensive star-formation takes place, this phenomenon might provide an explanation for the nature of MAGIC J1857.6+0297 near the PWN HESSJ1857+026.

\section{References}

[1] MAGIC Collaboration, J. Aleksic, S. Ansoldi, L. A. Antonelli, P. Antoranz, A. Babic, P. Bangale, M. Barcelo, J.A. Barrio, J. Becerra Gonzalez, et al., ArXiv e-prints (2014), 1409.5594

[2] J. Aleksić, E.A. Alvarez, L.A. Antonelli, P. Antoranz, M. Asensio, M. Backes, U. Barres de Almeida, J.A. Barrio, D. Bastieri, J. Becerra González, et al., A\&A 541, A13 (2012), 1201.4074

[3] MAGIC Collaboration, J. Aleksić, S. Ansoldi, L.A. Antonelli, P. Antoranz, A. Babic, P. Bangale, U. Barres de Almeida, J.A. Barrio, J. Becerra González, et al., A\&A 571, A96 (2014), 1401.7154

[4] J. Aleksić, E.A. Alvarez, L.A. Antonelli, P. Antoranz, M. Asensio, M. Backes, J.A. Barrio, D. Bastieri, J. Becerra González, W. Bednarek, et al., Astroparticle Physics 35, 435 (2012), 1108. 1477

[5] C.L. Brogan, W.M. Goss, T.R. Hunter, A.M.S. Richards, C.J. Chandler, J.S. Lazendic, B.C. Koo, I.M. Hoffman, M.J. Claussen, ApJ 771, 91 (2013), 1305.2793

[6] B.C. Koo, D.S. Moon, ApJ 475, 194 (1997)

[7] B.C. Koo, D.S. Moon, ApJ 485, 263 (1997)

[8] J.W. Hewitt, F. Yusef-Zadeh, M. Wardle, ApJ 683, 189 (2008), 0802.3878

[9] W.W. Tian, D.A. Leahy, ApJ 769, L17 (2013), 1305.0325

[10] B.C. Koo, K.T. Kim, F.D. Seward, ApJ 447, 211 (1995)

[11] B.C. Koo, J.J. Lee, F.D. Seward, AJ 123, 1629 (2002), arXiv:astro-ph/0112367

[12] B.C. Koo, J.J. Lee, F.D. Seward, D.S. Moon, ApJ 633, $946(2005)$

[13] M. Sasaki, C. Heinitz, G. Warth, G. Pühlhofer, A\&A 563, A9 (2014), 1401.8143

[14] Y. Hanabata, M. Sawada, H. Katagiri, A. Bamba, Y. Fukazawa, PASJ 65, 42 (2013), 1212. 1993

[15] A.A. Abdo, M. Ackermann, M. Ajello, L. Baldini, J. Ballet, G. Barbiellini, M.G. Baring, D. Bastieri, B.M. Baughman, K. Bechtol, et al., ApJ 706, L1 (2009), 0910.0908

[16] A. Fiasson, V. Marandon, R.C.G. Chaves, O. Tibolla, Discovery of a VHE gamma-ray source in the W51 region, in Proceedings of the 31st ICRC, Lodz (2009)

[17] A.A. Abdo, B.T. Allen, T. Aune, D. Berley, C. Chen, G.E. Christopher, T. DeYoung, B.L. Dingus, R.W. 
Ellsworth, M.M. Gonzalez, et al., ApJ 700, L127 (2009), 0904.1018

[18] T. Jogler, for the Fermi/LAT Collaboration, Revealing Cosmic-Ray acceleration in the SNR W51C, in these proceedings (2015)

[19] M. Ackermann, M. Ajello, A. Allafort, L. Baldini, J. Ballet, G. Barbiellini, M.G. Baring, D. Bastieri, K. Bechtol, R. Bellazzini, et al., Science 339, 807 (2013), 1302.3307

[20] D.S. Moon, B.C. Koo, Journal of Korean Astronomical Society 27, 81 (1994)

[21] F. Aharonian, A.G. Akhperjanian, U. Barres de Almeida, A.R. Bazer-Bachi, B. Behera, M. Beilicke, W. Benbow, K. Bernlöhr, C. Boisson, O. Bolz, et al., A\&A 477, 353 (2008), 0712.1173

[22] J.W.T. Hessels, D.J. Nice, B.M. Gaensler, V.M. Kaspi, D.R. Lorimer, D.J. Champion, A.G. Lyne, M. Kramer, J.M. Cordes, P.C.C. Freire, et al., ApJ 682, L41 (2008), 0806. 1200

[23] R. Rousseau, M.H. Grondin, A. Van Etten, M. Lemoine-Goumard, S. Bogdanov, J.W.T. Hessels, V.M. Kaspi, Z. Arzoumanian, F. Camilo, J.M.
Casandjian, et al., A\&A 544, A3 (2012), 1206. 3324

[24] J.M. Jackson, J.M. Rathborne, R.Y. Shah, R. Simon, T.M. Bania, D.P. Clemens, E.T. Chambers, A.M. Johnson, M. Dormody, R. Lavoie, et al., ApJs 163 , 145 (2006), arXiv: astro-ph/0602160

[25] J. Roman-Duval, J.M. Jackson, M. Heyer, A. Johnson, J. Rathborne, R. Shah, R. Simon, ApJ 699, 1153 (2009), 0905.0723

[26] O.C. de Jager, A. Djannati-Ataï, Implications of HESS Observations of Pulsar Wind Nebulae, in Astrophysics and Space Science Library, edited by W. Becker (2009), Vol. 357 of Astrophysics and Space Science Library, p. 451, 0803.0116

[27] A.T. Araudo, G.E. Romero, V. Bosch-Ramon, J.M. Paredes, International Journal of Modern Physics D 17, 1889 (2008), 0805.1898

[28] V. Bosch-Ramon, G.E. Romero, A.T. Araudo, J.M. Paredes, A\&A 511, A8 (2010), 0911.5612

[29] L.D. Anderson, T.M. Bania, J.M. Jackson, D.P. Clemens, M. Heyer, R. Simon, R.Y. Shah, J.M. Rathborne, ApJS 181, 255 (2009), 0810.3685 